\title{
Cover of Cylinder Lattice Plastic Convert into Fuel
}

\author{
Moinuddin Sarker*, Mohammad Mamunor Rashid \\ Department of Research and Development, Natural State Research, Inc., \\ 37 Brown House Road ( $2^{\text {nd }}$ Floor), Stamford, CT-06902, USA \\ Phone: (203) 406-0675, Fax: (203) 406-9852 \\ *E-mail address: msarker@naturalstateresearch.com , mamun_sarker2002@yahoo.com
}

\begin{abstract}
Waste plastic of cylinder lattice to liquid fuel production process was performing two step processes. $1^{\text {st }}$ step process was perform muffle furnace with ceramic crucible and $2^{\text {nd }}$ step process was perform glass reactor with condensation unit. Thermal degradation process was performing with furnace and temperature was $410{ }^{\circ} \mathrm{C}$ and reactor temperature was $420^{\circ} \mathrm{C}$. Muffle furnace was use for solid hard shape of plastic melting purpose and glass reactor was use for liquid slurry to produce vapor purpose. Liquid slurry to produce vapor was condensed and collected liquid fuel and fuel density is $0.75 \mathrm{~g} / \mathrm{ml}$. Liquid fuel production conversation rate was $71.05 \%$. Fuel was analysis by GC/MS and carbon chains obtain $\mathrm{C}_{3}$ to $\mathrm{C}_{23}$ from GC/MS chromatogram. Fuel color is light yellow and fuel is ignited.
\end{abstract}

Keywords: plastic lattice; conversion; fuel; hydrocarbon; waste plastic; GC/MS

\section{INTRODUCTION}

Every year large amounts of mixed plastic waste (MPW), mainly consisting of polyethylene (PE), polypropylene (PP), polystyrene (PS), and poly (vinyl chloride), (PVC) are produced. At the moment this waste is usually dumped or incinerated together with household waste, but due to environmental concerns, governments, companies, and universities are looking at alternatives for the disposal of this waste. One very promising alternative to dumping or incineration is high-temperature pyrolysis of the MPW to recover valuable chemicals, like ethene, propene, and styrene [1]. Recently, plastics recycling has received much attention all over the world because of serious environmental problems caused by waste plastics as well as their potential for use as resources. Landfill and incineration have not gained social acceptance as the methods for disposing of the waste, and they are becoming legally restricted because of strong pollution concerns. A chemical method that converts waste plastics into chemical resources or fuels is of great interest as an alternative because it provides a viable means of contributing to solution of the problems [2].

Degradable polyolefins have a long history. During the 1970s, a number of products based on polyethylene were commercialized. It was recognized at that time that polyolefins as produced were oxidatively unstable in the environment, and early investigations showed that the reason for their instability was the presence of sensitizing impurities in the polymer [3-6].

The most important of these were carbonyl $(>C=O)[3,5,6]$ and hydroperoxide groups $(-\mathrm{OOH})[3,7-10]$ formed during manufacture of plastics products. This led to extensive 
studies in the polymer industries and later in universities directed toward extending the lifetime of polymers by using heat and light stabilizers [7-14]. By contrast, the polyolefins had already achieved a central position in the distribution of consumer goods because of their combination of flexibility, toughness, and excellent barrier properties, which has made them the materials of choice for packaging applications [15]. They were particularly important in blown film technology and injection molding because of their ease of conversion and low cost. The present day efficient distribution of perishable foodstuffs is a direct consequence of the resistance of the polyolefins and other carbon-chain polymers to water and water-borne microorganisms [16], and in agriculture, the new technology of plasticulture based on polyethylene was already making an impact on the growing of soft fruits and vegetables [17, $18,19,20]$.

The various methods of plastic waste recycling [21], thermal and/or catalytic degradation of plastic waste to gas and liquid products are the most promising to be developed into a commercial polymer recycling process.

The products of such a process could be utilized as fuels or chemicals. These way waste plastics could be regarded as a cheap source of material. Because pure thermal degradation demands relatively high temperatures and its products require further processing for their quality to be upgraded, catalytic degradation of plastic waste offers considerable advantages [21-31].

There has been considerable work conducted on pyrolysis of polymers, some of which aimed at pyrolytic recycling of plastics to monomers and fuels. Thermal conversion of plastics, both pyrolysis and gasification, has been extensively studied, and commercial processes have been developed [32, 33] to convert waste plastics to fuels. However, very few works focused on hydrogen as the main product from waste plastics [34]. studied a fluidized bed co-gasification of coal, biomass, and plastics to generate hydrogen-rich gas [35]. Patented a process for manufacturing synthesis gas from petroleum residues and waste plastics. Recently [36, 37], reported on catalytic steam reforming of oils produced by pyrolysis of plastics at $350-400{ }^{\circ} \mathrm{C}$.

\section{MATERIALS AND METHOD}

Waste material cover of cylinder lattice was collected from helium gas cylinder and it was hard transparent. When helium gas was order for laboratory that time it was coming with helium gas cylinder as a cover. Usually this cover was throughout as a waste plastic. Cover of cylinder lattice was cut into small pieces and small pieces cylinder lattice was placed into crucible for muffle furnace. Muffle furnace provided company was Barnstead International and model F 6000. Full muffle furnace placed was Labconco fume hood and model number is 6910110 . Muffle furnace temperature capable up to $1400^{\circ} \mathrm{C}$, but out program temperature was $430{ }^{\circ} \mathrm{C}$ and temperature ramping rate was $20^{\circ} \mathrm{C}$ per minute. Waste plastic and crucible was placed into muffle furnace inside and covered the muffle furnace door.

Total solid sample was $250.8 \mathrm{gm}$. Then start the muffle furnace for solid plastic to melt at $410{ }^{\circ} \mathrm{C}$ for 21 minutes. When temperature was researched at $410^{\circ} \mathrm{C}$ then temperature was hold for 20 minutes. After 20 minutes running muffle furnace temperature goes down $5^{\circ} \mathrm{C}$ per minute. When temperature was down $150{ }^{\circ} \mathrm{C}$ then muffle furnace door was open and took out crucible with crucible cover and placed into fume hood inside. Then liquid slurry was placed into glass reactor inside from crucible. During this process solid sample was liquid and some solid sample was vapor and come out outside. 


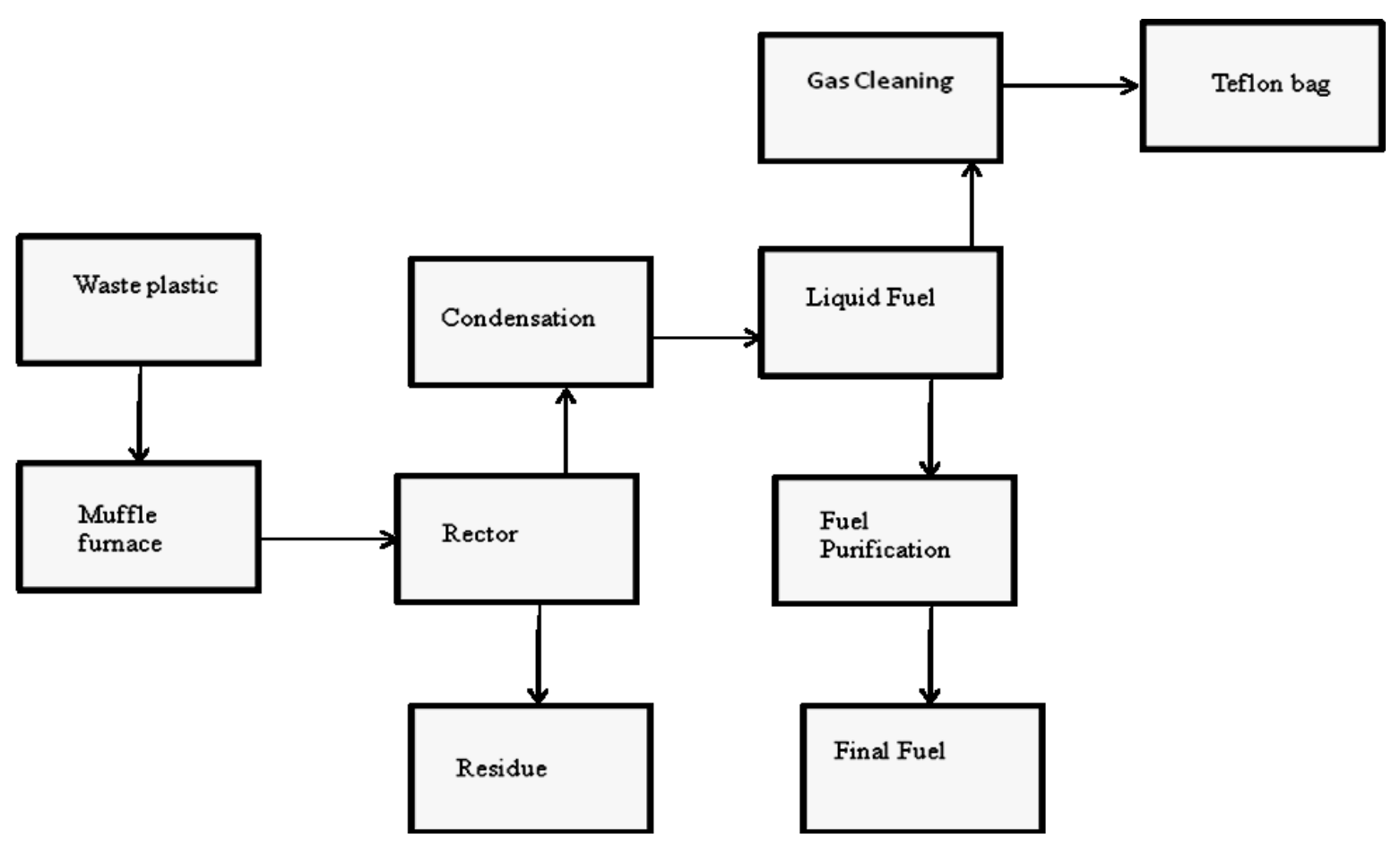

Figure 1. Cover of cylinder lattice to fuel production process step.

Fume hood Hepa filter was absorbed produce vapor because condensation was not setup with muffle furnace. Our experimental main goal was cylinder lattice to fuel production process with muffle furnace through glass reactor. During this process none of smoke or vapor did not come outside and all vapors was absorbed by fume hood filter. Muffle furnace liquefaction process solid sample to liquid slurry period produce vapor percentage was $7.53 \%$ $(18.9 \mathrm{gm})$. This process was $1^{\text {st }}$ step process and $2^{\text {nd }}$ step process was liquid slurry to produce liquid hydrocarbon fuel (Figure 1). When liquid waste plastic slurry transfer into glass reactor inside manually and liquid slurry weight was $231.9 \mathrm{gm}$, then glass reactor was heated up to $420{ }^{\circ} \mathrm{C}$ and temperature was monitored by variac meter. Condensation was setup with reactor but cooling water did not use as a condensation process. Room temperature was enough for produce vapor condensation purpose. Waste plastic liquid slurry was heated up continually until finish the full slurry to produce vapor and collected liquid fuel. Liquid slurry to fuel production period also light gas was generated and light gas was treated with alkali solution to remove contamination.

Fuel was cleaned with micron filter and clean fuel keeps into separate container for future use or analysis. Liquid fuel was recovering $178.2 \mathrm{gm}$ (volume $235 \mathrm{ml}$ ), left over residue was $25 \mathrm{gm}$, light gas was generated from sample $15 \mathrm{gm}$, and $13.7 \mathrm{gm}$ was stuck with crucible inside surface. Liquid fuel recover percentage was $71.05 \%$. Cover of cylinder lattice was non coded waste plastic and produce residue was solid hard and black color. Product fuel color is light yellow and fuel is ignited. Product fuel density is $0.75 \mathrm{~g} / \mathrm{ml}$. After finished the experiment solid black residue take out form glass reactor and keep for separate container. 


\section{RESULTS AND DISCUSSION}

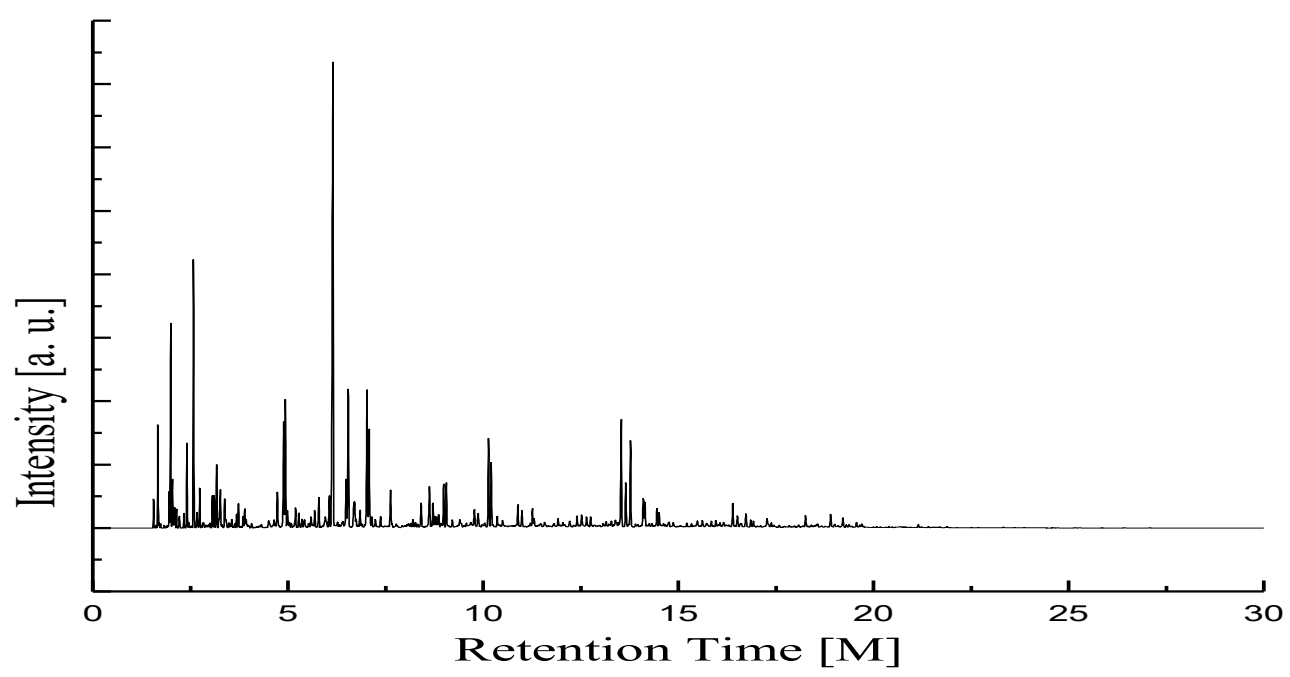

Figure 2. GC/MS chromatogram of cover of cylinder lattice to fuel.

Table 1. GC/MS chromatogram compounds list of cover of cylinder lattice to fuel.

\begin{tabular}{|c|c|c|c|c|c|c|c|}
\hline $\begin{array}{l}\text { Number } \\
\text { of Peak }\end{array}$ & $\begin{array}{c}\text { Reten- } \\
\text { tion } \\
\text { Time } \\
\text { (min.) }\end{array}$ & $\begin{array}{l}\text { Trace } \\
\text { Mass } \\
(\mathbf{m} / \mathbf{z})\end{array}$ & $\begin{array}{l}\text { Compound } \\
\text { Name }\end{array}$ & $\begin{array}{l}\text { Compound } \\
\text { Formula }\end{array}$ & $\begin{array}{c}\text { Mole- } \\
\text { cular } \\
\text { Weight }\end{array}$ & $\begin{array}{l}\text { Probabil } \\
\text { ity } \%\end{array}$ & $\begin{array}{c}\text { NIST Library } \\
\text { Number }\end{array}$ \\
\hline 1 & 1.56 & 41 & Cyclopropane & $\mathrm{C}_{3} \mathrm{H}_{6}$ & 42 & 50.1 & 18854 \\
\hline 2 & 1.66 & 41 & 2-methyl-propene & $\mathrm{C}_{4} \mathrm{H}_{8}$ & 56 & 33.9 & 18910 \\
\hline 3 & 1.95 & 42 & ethyl-cyclopropane & $\mathrm{C}_{5} \mathrm{H}_{10}$ & 70 & 21.7 & 114410 \\
\hline 4 & 2.00 & 43 & pentane & $\mathrm{C}_{5} \mathrm{H}_{12}$ & 72 & 79.0 & 114462 \\
\hline 5 & 2.04 & 55 & $\begin{array}{l}\text { trans-1,2-dimethyl- } \\
\text { cyclopropane }\end{array}$ & $\mathrm{C}_{5} \mathrm{H}_{10}$ & 70 & 13.5 & 114453 \\
\hline 6 & 2.08 & 55 & $\begin{array}{l}\text { cis-1,2-dimethyl- } \\
\text { cyclopropane, }\end{array}$ & $\mathrm{C}_{5} \mathrm{H}_{10}$ & 70 & 19.0 & 248 \\
\hline 7 & 2.10 & 55 & (E)-2-pentene & $\mathrm{C}_{5} \mathrm{H}_{10}$ & 70 & 17.3 & 291780 \\
\hline
\end{tabular}




\begin{tabular}{|c|c|c|c|c|c|c|c|}
\hline 8 & 2.15 & 67 & 1,3-pentadiene & $\mathrm{C}_{5} \mathrm{H}_{8}$ & 68 & 17.3 & 291890 \\
\hline 9 & 2.22 & 67 & (Z)-1,3-pentadiene & $\mathrm{C}_{5} \mathrm{H}_{8}$ & 68 & 15.5 & 211 \\
\hline 10 & 2.34 & 41 & 1-Pentene, 4-methyl- & $\mathrm{C}_{6} \mathrm{H}_{12}$ & 84 & 17.2 & 149350 \\
\hline 11 & 2.41 & 43 & Pentane, 2-methyl- & $\mathrm{C}_{6} \mathrm{H}_{14}$ & 86 & 65.0 & 61279 \\
\hline 12 & 2.58 & 56 & 1-Pentene, 2-methyl- & $\mathrm{C}_{6} \mathrm{H}_{12}$ & 84 & 33.9 & 495 \\
\hline 13 & 2.67 & 57 & Hexane & $\mathrm{C}_{6} \mathrm{H}_{14}$ & 86 & 76.6 & 61280 \\
\hline 14 & 2.74 & 41 & 2-Butene, 2,3-dimethyl- & $\mathrm{C}_{6} \mathrm{H}_{12}$ & 84 & 16.0 & 289588 \\
\hline 15 & 3.06 & 67 & 2,4-Hexadiene, (Z,Z)- & $\mathrm{C}_{6} \mathrm{H}_{10}$ & 82 & 12.6 & 113646 \\
\hline 16 & 3.17 & 56 & 1-Pentene, 2,4-dimethyl- & $\mathrm{C}_{7} \mathrm{H}_{14}$ & 98 & 64.0 & 114435 \\
\hline 17 & 3.27 & 81 & $\begin{array}{l}\text { 2,4-Dimethyl 1,4- } \\
\text { pentadiene }\end{array}$ & $\mathrm{C}_{7} \mathrm{H}_{12}$ & 96 & 44.8 & 114468 \\
\hline 18 & 3.38 & 78 & Benzene & $\mathrm{C}_{6} \mathrm{H}_{6}$ & 78 & 69.9 & 114388 \\
\hline 19 & 3.56 & 81 & 1,5-Hexadiene, 2-methyl- & $\mathrm{C}_{7} \mathrm{H}_{12}$ & 96 & 48.3 & 114394 \\
\hline 20 & 3.69 & 56 & 1-Hexene, 2-methyl- & $\mathrm{C}_{7} \mathrm{H}_{14}$ & 98 & 42.5 & 114433 \\
\hline 21 & 3.73 & 41 & 1-Heptene & $\mathrm{C}_{7} \mathrm{H}_{14}$ & 98 & 34.4 & 107734 \\
\hline 22 & 3.85 & 43 & Heptane & $\mathrm{C}_{7} \mathrm{H}_{16}$ & 100 & 46.1 & 61276 \\
\hline 23 & 3.89 & 81 & $\begin{array}{l}\text { 1,3-Pentadiene, 2,4- } \\
\text { dimethyl- }\end{array}$ & $\mathrm{C}_{7} \mathrm{H}_{12}$ & 96 & 19.8 & 114450 \\
\hline 24 & 3.93 & 81 & $\begin{array}{l}\text { Cyclopentene, 4,4- } \\
\text { dimethyl- }\end{array}$ & $\mathrm{C}_{7} \mathrm{H}_{12}$ & 96 & 12.8 & 38642 \\
\hline
\end{tabular}




\begin{tabular}{|c|c|c|c|c|c|c|c|}
\hline 25 & 4.07 & 81 & $\begin{array}{l}\text { Cyclopropane, 1,1,2- } \\
\text { trimethyl-3-methylene- }\end{array}$ & $\mathrm{C}_{7} \mathrm{H}_{12}$ & 96 & 9.54 & 63085 \\
\hline 26 & 4.13 & 79 & $\begin{array}{l}\text { 1,3-Cyclopentadiene, 5,5- } \\
\text { dimethyl- }\end{array}$ & $\mathrm{C}_{7} \mathrm{H}_{10}$ & 94 & 28.6 & 161866 \\
\hline 27 & 4.21 & 67 & 1-Ethylcyclopentene & $\mathrm{C}_{7} \mathrm{H}_{12}$ & 96 & 36.6 & 114407 \\
\hline 28 & 4.28 & 67 & $\begin{array}{l}\text { Cyclopropane, 1-(1,1- } \\
\text { dimethylethyl)-2- } \\
\text { methylene- }\end{array}$ & $\mathrm{C}_{8} \mathrm{H}_{14}$ & 110 & 4.16 & 62490 \\
\hline 29 & 4.32 & 81 & $\begin{array}{l}\text { 1-Ethyl-5- } \\
\text { methylcyclopentene }\end{array}$ & $\mathrm{C}_{8} \mathrm{H}_{14}$ & 110 & 8.36 & 114420 \\
\hline 30 & 4.42 & 41 & Cyclopentane, ethyl- & $\mathrm{C}_{7} \mathrm{H}_{14}$ & 98 & 10.7 & 940 \\
\hline 31 & 4.51 & 79 & $\begin{array}{l}\text { 2,4-Heptadien-1-ol, } \\
(\mathrm{E}, \mathrm{E})-\end{array}$ & $\mathrm{C}_{7} \mathrm{H}_{12} \mathrm{O}$ & 112 & 31.1 & 1645 \\
\hline 32 & 4.64 & 56 & 2,4-Dimethyl-1-hexene & $\mathrm{C}_{8} \mathrm{H}_{16}$ & 112 & 47.1 & 113443 \\
\hline 33 & 4.73 & 69 & 2-Hexene, 3,5-dimethyl- & $\mathrm{C}_{8} \mathrm{H}_{16}$ & 112 & 11.9 & 149385 \\
\hline 34 & 4.89 & 43 & Heptane, 4-methyl- & $\mathrm{C}_{8} \mathrm{H}_{18}$ & 114 & 63.9 & 113916 \\
\hline 35 & 4.93 & 91 & Toluene & $\mathrm{C}_{7} \mathrm{H}_{8}$ & 92 & 50.8 & 291301 \\
\hline 36 & 4.99 & 55 & $\begin{array}{l}\text { 1,4-Hexadiene, } 2,5- \\
\text { dimethyl- }\end{array}$ & $\mathrm{C}_{8} \mathrm{H}_{14}$ & 110 & 7.99 & 61785 \\
\hline 37 & 5.20 & 56 & 1-Heptene, 2-methyl- & $\mathrm{C}_{8} \mathrm{H}_{16}$ & 112 & 53.0 & 113675 \\
\hline 38 & 5.28 & 55 & 2-Octene & $\mathrm{C}_{8} \mathrm{H}_{16}$ & 112 & 10.1 & 118191 \\
\hline 39 & 5.36 & 95 & $\begin{array}{l}\text { 5,5-Dimethyl-1,3- } \\
\text { hexadiene }\end{array}$ & $\mathrm{C}_{8} \mathrm{H}_{14}$ & 110 & 14.4 & 142720 \\
\hline 40 & 5.43 & 43 & Octane & $\mathrm{C}_{8} \mathrm{H}_{18}$ & 114 & 28.3 & 229407 \\
\hline 41 & 5.69 & 83 & $\begin{array}{l}\text { Cyclopentane, 1,1,3,4- } \\
\text { tetramethyl-, cis- }\end{array}$ & $\mathrm{C}_{9} \mathrm{H}_{18}$ & 126 & 12.2 & 27589 \\
\hline
\end{tabular}




\begin{tabular}{|c|c|c|c|c|c|c|c|}
\hline 42 & 5.79 & 43 & Heptane, 2,4-dimethyl- & $\mathrm{C}_{9} \mathrm{H}_{20}$ & 128 & 32.2 & 155382 \\
\hline 43 & 6.06 & 69 & $\begin{array}{l}\text { Cyclohexane, } 1,3,5- \\
\text { trimethyl- }\end{array}$ & $\mathrm{C}_{9} \mathrm{H}_{18}$ & 126 & 46.1 & 114702 \\
\hline 44 & 6.15 & 70 & 2,4-Dimethyl-1-heptene & $\mathrm{C}_{9} \mathrm{H}_{18}$ & 126 & 49.4 & 113516 \\
\hline 45 & 6.49 & 69 & $\begin{array}{l}\text { Cyclohexane, } 1,3,5- \\
\text { trimethyl-, }(1 \alpha, 3 \alpha, 5 \beta)-\end{array}$ & $\mathrm{C}_{9} \mathrm{H}_{18}$ & 126 & 36.2 & 2480 \\
\hline 46 & 6.54 & 91 & Ethylbenzene & $\mathrm{C}_{8} \mathrm{H}_{10}$ & 106 & 54.0 & 158804 \\
\hline 47 & 6.71 & 91 & $\begin{array}{l}\text { Cyclohexene, 3,3,5- } \\
\text { trimethyl- }\end{array}$ & $\mathrm{C}_{9} \mathrm{H}_{16}$ & 124 & 30.0 & 114765 \\
\hline 48 & 6.85 & 109 & $\begin{array}{l}\text { Cyclohexene, } 3,3,5- \\
\text { trimethyl- }\end{array}$ & $\mathrm{C}_{9} \mathrm{H}_{16}$ & 124 & 37.3 & 114765 \\
\hline 49 & 7.03 & 43 & $\begin{array}{l}\text { 2-Pentanone, 3- } \\
{[(\text { acetyloxy)methyl]-3,4- }} \\
\text { dimethyl-, (.土.)- }\end{array}$ & $\begin{array}{c}\mathrm{C}_{10} \mathrm{H}_{18} \mathrm{O} \\
3\end{array}$ & 186 & 7.58 & 186591 \\
\hline 50 & 7.08 & 104 & Styrene & $\mathrm{C}_{8} \mathrm{H}_{8}$ & 104 & 40.2 & 291542 \\
\hline 51 & 7.15 & 43 & Hexane, 2,4-dimethyl- & $\mathrm{C}_{8} \mathrm{H}_{18}$ & 114 & 9.02 & 118871 \\
\hline 52 & 7.24 & 83 & $\begin{array}{l}\text { Bicyclo[3.1.1]heptan-2- } \\
\text { one, 6,6-dimethyl-, (1R)- }\end{array}$ & $\mathrm{C}_{9} \mathrm{H}_{14} \mathrm{O}$ & 138 & 19.8 & 108460 \\
\hline 53 & 7.37 & 55 & $\begin{array}{l}\text { 1,6-Octadiene, 2,5- } \\
\text { dimethyl-, (E)- }\end{array}$ & $\mathrm{C}_{10} \mathrm{H}_{18}$ & 138 & 6.10 & 62075 \\
\hline 54 & 7.63 & 105 & Benzene, (1-methylethyl)- & $\mathrm{C}_{9} \mathrm{H}_{12}$ & 120 & 53.5 & 228742 \\
\hline 55 & 8.06 & 57 & $\begin{array}{l}\text { 2-Undecanethiol, 2- } \\
\text { methyl- }\end{array}$ & $\mathrm{C}_{12} \mathrm{H}_{26} \mathrm{~S}$ & 202 & 3.12 & 9094 \\
\hline 56 & 8.09 & 43 & cis-3-Decene & $\mathrm{C}_{10} \mathrm{H}_{20}$ & 140 & 16.4 & 113558 \\
\hline 57 & 8.15 & 91 & Benzene, propyl- & $\mathrm{C}_{9} \mathrm{H}_{12}$ & 120 & 47.6 & 113930 \\
\hline 58 & 8.20 & 57 & Nonane, 4-methyl- & $\mathrm{C}_{10} \mathrm{H}_{22}$ & 142 & 25.8 & 3834 \\
\hline
\end{tabular}




\begin{tabular}{|c|c|c|c|c|c|c|c|}
\hline 59 & 8.27 & 105 & $\begin{array}{l}\text { Benzene, 1-ethyl-3- } \\
\text { methyl- }\end{array}$ & $\mathrm{C}_{9} \mathrm{H}_{12}$ & 120 & 31.2 & 228743 \\
\hline 60 & 8.33 & 105 & 1-Decen-4-yne, 2-nitro- & $\begin{array}{c}\mathrm{C}_{10} \mathrm{H}_{15} \mathrm{~N} \\
2\end{array}$ & 181 & 9.03 & 186798 \\
\hline 61 & 8.41 & 94 & Phenol & $\mathrm{C}_{6} \mathrm{H}_{6} \mathrm{O}$ & 94 & 27.1 & 221160 \\
\hline 62 & 8.62 & 118 & $\alpha$-Methylstyrene & $\mathrm{C}_{9} \mathrm{H}_{10}$ & 118 & 34.8 & 229186 \\
\hline 63 & 8.72 & 69 & 3-Undecene, (Z)- & $\mathrm{C}_{11} \mathrm{H}_{22}$ & 154 & 5.05 & 142598 \\
\hline 64 & 8.76 & 69 & 3-Undecene, (Z)- & $\mathrm{C}_{11} \mathrm{H}_{22}$ & 154 & 5.19 & 142598 \\
\hline 65 & 8.81 & 69 & 4-Undecene, (Z)- & $\mathrm{C}_{11} \mathrm{H}_{22}$ & 154 & 4.99 & 142600 \\
\hline 66 & 8.86 & 57 & Valeric acid, 4-phenyl- & $\begin{array}{c}\mathrm{C}_{11} \mathrm{H}_{14} \mathrm{O} \\
2\end{array}$ & 178 & 36.0 & 99257 \\
\hline 67 & 8.92 & 57 & 1-Decene, 4-methyl- & $\mathrm{C}_{11} \mathrm{H}_{22}$ & 154 & 8.70 & 150275 \\
\hline 68 & 8.99 & 43 & Octane, 3,3-dimethyl- & $\mathrm{C}_{10} \mathrm{H}_{22}$ & 142 & 9.88 & 61706 \\
\hline 69 & 9.06 & 43 & Nonane, 2,6-dimethyl- & $\mathrm{C}_{11} \mathrm{H}_{24}$ & 156 & 10.7 & 61438 \\
\hline 70 & 9.21 & 43 & 4-Decene, 7-methyl-, (E)- & $\mathrm{C}_{11} \mathrm{H}_{22}$ & 154 & 7.95 & 60846 \\
\hline 71 & 9.40 & 55 & 3-Undecene, (E)- & $\mathrm{C}_{11} \mathrm{H}_{22}$ & 154 & 5.14 & 60565 \\
\hline 72 & 9.52 & 69 & $\begin{array}{l}\text { 2,3,4-Trimethyl-hex-3- } \\
\text { enal }\end{array}$ & $\mathrm{C}_{9} \mathrm{H}_{16} \mathrm{O}$ & 140 & 6.07 & 193729 \\
\hline 73 & 9.57 & 56 & 4-Undecene, $(Z)-$ & $\mathrm{C}_{11} \mathrm{H}_{22}$ & 154 & 4.74 & 142600 \\
\hline 74 & 9.78 & 43 & 1-Octanol, 2-butyl- & $\mathrm{C}_{12} \mathrm{H}_{26} \mathrm{O}$ & 186 & 4.27 & 114639 \\
\hline 75 & 9.87 & 43 & Undecane, 2-methyl- & $\mathrm{C}_{12} \mathrm{H}_{26}$ & 170 & 5.03 & 6605 \\
\hline
\end{tabular}




\begin{tabular}{|c|c|c|c|c|c|c|c|}
\hline 76 & 10.04 & 69 & $\begin{array}{l}\text { 4-Chloro-3-n- } \\
\text { hexyltetrahydropyran }\end{array}$ & $\begin{array}{c}\mathrm{C}_{11} \mathrm{H}_{21} \mathrm{Cl} \\
\mathrm{O}\end{array}$ & 204 & 5.89 & 216835 \\
\hline 77 & 10.14 & 69 & $\begin{array}{l}\text { 2-Undecanethiol, 2- } \\
\text { methyl- }\end{array}$ & $\mathrm{C}_{12} \mathrm{H}_{26} \mathrm{~S}$ & 202 & 4.49 & 9094 \\
\hline 78 & 10.21 & 43 & 1-Octanol, 2,7-dimethyl- & $\mathrm{C}_{10} \mathrm{H}_{22} \mathrm{O}$ & 158 & 2.89 & 5475 \\
\hline 79 & 10.36 & 55 & 3-Undecene, (Z)- & $\mathrm{C}_{11} \mathrm{H}_{22}$ & 154 & 4.06 & 142598 \\
\hline 80 & 10.50 & 57 & Undecane & $\mathrm{C}_{11} \mathrm{H}_{24}$ & 156 & 21.2 & 114185 \\
\hline 81 & 10.89 & 43 & $\begin{array}{l}\text { 1-Dodecanol, 3,7,11- } \\
\text { trimethyl- }\end{array}$ & $\mathrm{C}_{15} \mathrm{H}_{32} \mathrm{O}$ & 228 & 4.14 & 114065 \\
\hline 82 & 11.00 & 43 & $\begin{array}{l}\text { 1-Dodecanol, 3,7,11- } \\
\text { trimethyl- }\end{array}$ & $\mathrm{C}_{15} \mathrm{H}_{32} \mathrm{O}$ & 228 & 4.30 & 114065 \\
\hline 83 & 11.21 & 91 & $\begin{array}{l}\text { Benzene, (3-methyl-3- } \\
\text { butenyl)- }\end{array}$ & $\mathrm{C}_{11} \mathrm{H}_{14}$ & 146 & 26.7 & 27671 \\
\hline 84 & 11.27 & 69 & $\begin{array}{l}\text { (2,4,6- } \\
\text { Trimethylcyclohexyl) } \\
\text { methanol }\end{array}$ & $\mathrm{C}_{10} \mathrm{H}_{20} \mathrm{O}$ & 156 & 15.3 & 113757 \\
\hline 85 & 11.48 & 107 & 5,7-Dodecadiyn-1,12-diol & $\begin{array}{c}\mathrm{C}_{12} \mathrm{H}_{18} \mathrm{O} \\
2\end{array}$ & 194 & 35.5 & 136921 \\
\hline 86 & 11.58 & 69 & $\begin{array}{l}\text { 1-Isopropyl-1,4,5- } \\
\text { trimethylcyclohexane }\end{array}$ & $\mathrm{C}_{12} \mathrm{H}_{24}$ & 168 & 11.4 & 113584 \\
\hline 87 & 11.92 & 55 & 3-Dodecene, $(\mathrm{E})$ - & $\mathrm{C}_{12} \mathrm{H}_{24}$ & 168 & 5.52 & 113960 \\
\hline 88 & 12.22 & 69 & $\begin{array}{l}\text { 1-Dodecanol, 3,7,11- } \\
\text { trimethyl- }\end{array}$ & $\mathrm{C}_{15} \mathrm{H}_{32} \mathrm{O}$ & 228 & 9.68 & 114065 \\
\hline 89 & 12.41 & 121 & $\begin{array}{l}\text { Phenol, 4-(1- } \\
\text { methylethyl)- }\end{array}$ & $\mathrm{C}_{9} \mathrm{H}_{12} \mathrm{O}$ & 136 & 34.9 & 229733 \\
\hline 90 & 12.47 & 43 & 7-Hexadecenal, (Z)- & $\mathrm{C}_{16} \mathrm{H}_{30} \mathrm{O}$ & 238 & 12.0 & 293051 \\
\hline 91 & 12.53 & 57 & $\begin{array}{l}\text { Decane, } 2,3,5,8- \\
\text { tetramethyl- }\end{array}$ & $\mathrm{C}_{14} \mathrm{H}_{30}$ & 198 & 8.79 & 149589 \\
\hline 92 & 12.65 & 57 & Dodecane, 4,6-dimethyl- & $\mathrm{C}_{14} \mathrm{H}_{30}$ & 198 & 11.0 & 45335 \\
\hline
\end{tabular}




\begin{tabular}{|c|c|c|c|c|c|c|c|}
\hline 93 & 12.76 & 57 & $\begin{array}{l}\text { Decane, } 2,3,5,8- \\
\text { tetramethyl- }\end{array}$ & $\mathrm{C}_{14} \mathrm{H}_{30}$ & 198 & 9.12 & 149589 \\
\hline 94 & 13.15 & 57 & $\begin{array}{l}\text { Dodecane, } 2,6,10- \\
\text { trimethyl- }\end{array}$ & $\mathrm{C}_{15} \mathrm{H}_{32}$ & 212 & 6.66 & 114045 \\
\hline 95 & 13.29 & 43 & 2-Hexyl-1-octanol & $\mathrm{C}_{14} \mathrm{H}_{30} \mathrm{O}$ & 214 & 5.46 & 113807 \\
\hline 96 & 13.39 & 55 & 7-Tetradecene & $\mathrm{C}_{14} \mathrm{H}_{28}$ & 196 & 10.8 & 70643 \\
\hline 97 & 13.54 & 69 & $\begin{array}{l}\text { 1-Nonene, } 4,6,8- \\
\text { trimethyl- }\end{array}$ & $\mathrm{C}_{12} \mathrm{H}_{24}$ & 168 & 3.90 & 6413 \\
\hline 98 & 13.78 & 43 & $\begin{array}{l}\text { 1-Nonene, } 4,6,8- \\
\text { trimethyl- }\end{array}$ & $\mathrm{C}_{12} \mathrm{H}_{24}$ & 168 & 4.45 & 6413 \\
\hline 99 & 14.10 & 91 & Benzenebutanenitrile & $\mathrm{C}_{10} \mathrm{H}_{11} \mathrm{~N}$ & 145 & 88.6 & 236852 \\
\hline 100 & 14.15 & 43 & $\begin{array}{l}\text { 2-Isopropyl-5-methyl-1- } \\
\text { heptanol }\end{array}$ & $\mathrm{C}_{11} \mathrm{H}_{24} \mathrm{O}$ & 172 & 4.15 & 245029 \\
\hline 101 & 14.51 & 55 & 1,19-Eicosadiene & $\mathrm{C}_{20} \mathrm{H}_{38}$ & 278 & 6.45 & 158339 \\
\hline 102 & 14.87 & 57 & Tetradecane & $\mathrm{C}_{14} \mathrm{H}_{30}$ & 198 & 6.60 & 113925 \\
\hline 103 & 15.62 & 57 & $\begin{array}{l}\text { Decane, } 2,3,5,8- \\
\text { tetramethyl- }\end{array}$ & $\mathrm{C}_{14} \mathrm{H}_{30}$ & 198 & 10.2 & 149589 \\
\hline 104 & 15.72 & 57 & $\begin{array}{l}\text { Decane, } 2,3,5,8 \text { - } \\
\text { tetramethyl- }\end{array}$ & $\mathrm{C}_{14} \mathrm{H}_{30}$ & 198 & 11.5 & 149589 \\
\hline 105 & 16.07 & 55 & 1-Nonadecene & $\mathrm{C}_{19} \mathrm{H}_{38}$ & 266 & 4.05 & 113626 \\
\hline 106 & 16.16 & 57 & Nonadecane & $\mathrm{C}_{19} \mathrm{H}_{40}$ & 268 & 11.4 & 114098 \\
\hline 107 & 16.40 & 69 & $\begin{array}{l}\text { Trichloroacetic acid, } \\
\text { hexadecyl ester }\end{array}$ & $\begin{array}{c}\mathrm{C}_{18} \mathrm{H}_{33} \mathrm{Cl} \\
{ }_{3} \mathrm{O}_{2}\end{array}$ & 386 & 5.00 & 280518 \\
\hline 108 & 16.52 & 69 & 1-Decanol, 2-hexyl- & $\mathrm{C}_{16} \mathrm{H}_{34} \mathrm{O}$ & 242 & 5.39 & 114709 \\
\hline 109 & 17.28 & 69 & Cyclododecanemethanol & $\mathrm{C}_{13} \mathrm{H}_{26} \mathrm{O}$ & 198 & 8.87 & 108275 \\
\hline
\end{tabular}




\begin{tabular}{|c|c|c|l|c|c|c|c|}
\hline 110 & 18.26 & 92 & $\begin{array}{l}\text { Benzene, 1,1'-(1,3- } \\
\text { propanediyl)bis- }\end{array}$ & $\mathrm{C}_{15} \mathrm{H}_{16}$ & 196 & 95.1 & 229725 \\
\hline 111 & 18.57 & 57 & $\begin{array}{l}\text { Benzeneacetic acid, 4- } \\
\text { pentadecyl ester }\end{array}$ & $\begin{array}{c}\mathrm{C}_{23} \mathrm{H}_{38} \mathrm{O} \\
2\end{array}$ & 346 & 21.1 & 282028 \\
\hline 112 & 19.30 & 57 & $\begin{array}{l}\text { 1-Hexadecanol, } \\
3,7,11,15-\text { tetramethyl- }\end{array}$ & $\mathrm{C}_{20} \mathrm{H}_{42} \mathrm{O}$ & 298 & 4.30 & 194527 \\
\hline 113 & 21.70 & 57 & Nonadecane & $\mathrm{C}_{19} \mathrm{H}_{40}$ & 268 & 6.42 & 114098 \\
\hline
\end{tabular}

Waste cover of cylinder lattice to fuel product was analysis by gas chromatography and mass spectrometer (GC/MS) with auto sampler (Perkin Elmer Model Clarus 500). Fuel chromatogram and compounds data table showed figure 2 and table 1. Fuel compounds was detected from NIST library based on compound retention time (M) and trace mass $(\mathrm{m} / \mathrm{z})$. For compounds detection standard was follow restek hydrocarbon standard. GC/MS analysis purpose was use $\mathrm{CS}_{2}$ solution to clean the injection syringe before sample and after sample injected into $\mathrm{GC} / \mathrm{MS}$. GC/MS inject port temperature was $300{ }^{\circ} \mathrm{C}$ and program temperature was $250{ }^{\circ} \mathrm{C}$. GC/MS initial temperature was $40{ }^{\circ} \mathrm{C}$ and carrier gas was Helium. GC/MS column was elite capillary and length was 300 meter.

Product fuel GC/MS chromatogram was analysis including compounds name, compounds formula, compounds molecular weight, compounds probability percentage, compounds retention time, and compounds trace mass.

Liquid fuel analysis main goal was what types of compounds present inside fuel and how long carbon chains are present inside the fuel. Product fuel analysis compounds table showed chain showed $\mathrm{C}_{3}$ to $\mathrm{C}_{23}$. All compounds are not hydrocarbon compounds, analysis compounds table indicate that inside fuel has hydrocarbon compounds including alkane, alkene and alkyl group compounds.

Aromatic group related compounds, alcoholic compounds, oxygen content compounds, nitro group compounds and also halogen content compounds are present inside the fuel. Compounds are appeared such as Cyclopropane $\left(\mathrm{C}_{3} \mathrm{H}_{6}\right)(\mathrm{t}=1.56, \mathrm{~m} / \mathrm{z}=41), 2$-methyl-1Propene $\left(\mathrm{C}_{4} \mathrm{H}_{8}\right) \quad(\mathrm{t}=1.66, \mathrm{~m} / \mathrm{z}=41)$, cis-1,2-dimethyl- Cyclopropane $\left(\mathrm{C}_{5} \mathrm{H}_{10}\right) \quad(\mathrm{t}=2.08$, $\mathrm{m} / \mathrm{z}=55)$, 2-methyl-Pentane $\left(\mathrm{C}_{6} \mathrm{H}_{14}\right)(\mathrm{t}=2.41, \mathrm{~m} / \mathrm{z}=43)$, 2,3-dimethyl-2-Butene $\left(\mathrm{C}_{6} \mathrm{H}_{12}\right)$ $(\mathrm{t}=2.74, \mathrm{~m} / \mathrm{z}=41), 2$-methyl-1-Hexene $\left(\mathrm{C}_{7} \mathrm{H}_{14}\right)(\mathrm{t}=3.69, \mathrm{~m} / \mathrm{z}=56), 4,4$-dimethyl-Cyclopentene $\left(\mathrm{C}_{7} \mathrm{H}_{12}\right)(\mathrm{t}=3.93, \mathrm{~m} / \mathrm{z}=81)$, 1-Ethyl-5-methylcyclopentene $\left(\mathrm{C}_{8} \mathrm{H}_{14}\right)(\mathrm{t}=4.32, \mathrm{~m} / \mathrm{z}=81), 4-$ methyl-Heptane $\left(\mathrm{C}_{8} \mathrm{H}_{18}\right)(\mathrm{t}=4.89, \mathrm{~m} / \mathrm{z}=43)$, 2-methyl-1-Heptene $\left(\mathrm{C}_{8} \mathrm{H}_{16}\right)(\mathrm{t}=5.20, \mathrm{~m} / \mathrm{z}=56)$, cis-1,1,3,4-tetramethyl-Cyclopentane $\left(\mathrm{C}_{\mathrm{H}} 18\right)(\mathrm{t}=5.69, \mathrm{~m} / \mathrm{z}=83),(1 \alpha, 3 \alpha, 5 \beta)-1,3,5$-trimethylCyclohexane $\left(\mathrm{C}_{9} \mathrm{H}_{18}\right) \quad(\mathrm{t}=6.49, \mathrm{~m} / \mathrm{z}=69), 3,3,5$-trimethyl-Cyclohexene $\left(\mathrm{C}_{4} \mathrm{H}_{16}\right)(\mathrm{t}=6.85$, $\mathrm{m} / \mathrm{z}=109), 2,4$-dimethyl-Hexane $\left(\mathrm{C}_{8} \mathrm{H}_{18}\right)(\mathrm{t}=7.15, \mathrm{~m} / \mathrm{z}=43)$, cis-3-Decene $\left(\mathrm{C}_{10} \mathrm{H}_{20}\right)(\mathrm{t}=8.09$, $\mathrm{m} / \mathrm{z}=43), 2$-nitro-1-Decen-4-yn $\left(\mathrm{C}_{10} \mathrm{H}_{15} \mathrm{~N}_{2}\right)(\mathrm{t}=8.33, \mathrm{~m} / \mathrm{z}=105),(\mathrm{Z})-3-$ Undecene $\left(\mathrm{C}_{11} \mathrm{H}_{22}\right)$ $(\mathrm{t}=8.72, \mathrm{~m} / \mathrm{z}=69)$, 4-methyl-1-Decene $\left(\mathrm{C}_{11} \mathrm{H}_{22}\right)(\mathrm{t}=8.92, \mathrm{~m} / \mathrm{z}=57),(\mathrm{E})-7-$ methyl-4-Decene $\left(\mathrm{C}_{11} \mathrm{H}_{22}\right) \quad(\mathrm{t}=9.21, \mathrm{~m} / \mathrm{z}=43),(\mathrm{Z})-4-$ Undecene $\left(\mathrm{C}_{11} \mathrm{H}_{22}\right) \quad(\mathrm{t}=9.57, \mathrm{~m} / \mathrm{z}=56), 2$-methyl-2Undecanethiol $\left(\mathrm{C}_{12} \mathrm{H}_{26} \mathrm{~S}\right)(\mathrm{t}=10.14, \mathrm{~m} / \mathrm{z}=69), 3,7,11$-trimethyl-1-Dodecanol $\left(\mathrm{C}_{15} \mathrm{H}_{32} \mathrm{O}\right)$ $(\mathrm{t}=11.00, \mathrm{~m} / \mathrm{z}=43), \quad(\mathrm{Z})-7-H e x a d e c e n a l \quad\left(\mathrm{C}_{16} \mathrm{H}_{30} \mathrm{O}\right) \quad(\mathrm{t}=12.47, \mathrm{~m} / \mathrm{z}=43), \quad 4,6$-dimethylDodecane $\left(\mathrm{C}_{14} \mathrm{H}_{30}\right)(\mathrm{t}=12.65, \mathrm{~m} / \mathrm{z}=57), 2$-Hexyl-1-octanol $\left(\mathrm{C}_{14} \mathrm{H}_{30} \mathrm{O}\right)(\mathrm{t}=13.29, \mathrm{~m} / \mathrm{z}=43)$, 
4,6,8-trimethyl-1-Nonene $\left(\mathrm{C}_{12} \mathrm{H}_{24}\right)(\mathrm{t}=13.78, \mathrm{~m} / \mathrm{z}=43), 1,19$-Eicosadiene $\left(\mathrm{C}_{20} \mathrm{H}_{38}\right)(\mathrm{t}=14.51$, $\mathrm{m} / \mathrm{z}=55), 2,3,5,8$-tetramethyl-Decane $\left(\mathrm{C}_{14} \mathrm{H}_{30}\right)(\mathrm{t}=15.72, \mathrm{~m} / \mathrm{z}=57)$, Nonadecane $\left(\mathrm{C}_{19} \mathrm{H} 40\right)$ $(\mathrm{t}=16.16, \mathrm{~m} / \mathrm{z}=57), 4$-pentadecyl ester Benzeneacetic acid $\left(\mathrm{C}_{23} \mathrm{H}_{38} \mathrm{O}_{2}\right)(\mathrm{t}=18.57, \mathrm{~m} / \mathrm{z}=57)$ and so on.

Fuel chromatogram analysis compounds table showed also fuel has aromatic group compounds with different retention time such as Benzene $\left(\mathrm{C}_{6} \mathrm{H}_{6}\right)(\mathrm{t}=3.38, \mathrm{~m} / \mathrm{z}=78)$, Toluene $\left(\mathrm{C}_{7} \mathrm{H}_{8}\right)(\mathrm{t}=4.93, \mathrm{~m} / \mathrm{z}=91)$, Ethylbenzene $\left(\mathrm{C}_{8} \mathrm{H}_{10}\right)(\mathrm{t}=6.54, \mathrm{~m} / \mathrm{z}=91)$, Styrene $\left(\mathrm{C}_{8} \mathrm{H}_{8}\right)(\mathrm{t}=7.08$, $\mathrm{m} / \mathrm{z}=104)$, (1-methylethyl)-Benzene $\left(\mathrm{C}_{1} \mathrm{H}_{12}\right)(\mathrm{t}=7.63, \mathrm{~m} / \mathrm{z}=105)$, propyl-Benzene $\left(\mathrm{C}_{9} \mathrm{H}_{12}\right)$ $(\mathrm{t}=8.15, \mathrm{~m} / \mathrm{z}=91), 1$-ethyl-3-methyl- Benzene $\left(\mathrm{C}_{9} \mathrm{H}_{12}\right)(\mathrm{t}=8.27, \mathrm{~m} / \mathrm{z}=105)$, Phenol $\left(\mathrm{C}_{6} \mathrm{H}_{6} \mathrm{O}\right)$ $(\mathrm{t}=8.41, \quad \mathrm{~m} / \mathrm{z}=94), \quad \alpha$-Methylstyrene $\left(\mathrm{C}_{\mathrm{H}} \mathrm{H}_{10}\right) \quad(\mathrm{t}=8.62, \mathrm{~m} / \mathrm{z}=118), \quad$ (3-methyl-3-butenyl)Benzene $\left(\mathrm{C}_{11} \mathrm{H}_{14}\right) \quad(\mathrm{t}=11.21, \mathrm{~m} / \mathrm{z}=91), \quad 4-(1-$-methylethyl $)-P h e n o l \quad\left(\mathrm{C}_{9} \mathrm{H}_{12} \mathrm{O}\right) \quad(\mathrm{t}=12.42$, $\mathrm{m} / \mathrm{z}=121)$, and etc.

Waste cover of cylinder lattice was non coded waste plastic and after conversion GC/MS analysis result showed plastic to fuel has aromatic group compounds. So far we knew that these types of aromatic group compounds are present into polystyrene plastic.

\section{CONCLUSION}

Waste cover of cylinder lattice plastic to fuel recovery was successfully at temperature $420^{\circ} \mathrm{C}$. Muffle furnace through glass reactor was use for this experiment. Two step manual process experiment light gas generation percentage was $13.51 \%$ and liquid fuel production percentage was $71.05 \%$. Product fuel was analysis by GC/MS and compounds found that hydrocarbon including aromatic, alcoholic, and nitrogen content. None coded waste plastic cylinder lattice to fuel GC/MS analysis result showed that fuel has aromatic group compounds. Aromatic group compounds indicate that cylinder lattice plastic is look like polystyrene group plastic, because polystyrene plastic has aromatic group. Due to aromatic group present in the product fuel it can increase fuel efficiency. Product fuel has long chain hydrocarbon compounds for that reason it can use as diesel fuel.

By using this process non coded waste plastic can convert into liquid fuel and it can use for internal combustion engines. Waste plastic problem are everywhere and this technology can solve the waste plastic problem from environment.

\section{Acknowledgement}

The authors acknowledge the support (financial) of Dr. Karin Kaufman, the founder and sole owner of Natural State Research, Inc. The author also acknowledges the valuable contributions NSR laboratory team members during the preparation of this manuscript. 


\section{References}

[1] R. W. J. Westerhout, J. Waanders, J. A. M. Kuipers, W. P. M. van Swaaij, Ind. Eng. Chem. Res. 37 (1998) 2293-2300.

[2] Yoshio Uemichi, Junko Nakamura, Toshihiro Itoh, Masatoshi Sugioka, Ind. Eng. Chem. Res. 38 (1999) 385-390.

[3] Scott G., Atmospheric Oxidation and Antioxidants, Elsevier: Amsterdam 1965, pp. 272-282.

[4] Carlsson D. J., Wiles D. M., Encyclopedia of Polymer Science and Engineering, 2nd ed.; Wiley: New York 1986; Vol. 4, pp 631-696 and references therein.

[5] Guillet J. E., Norrish R. G. W., Nature 173 (1954) 625-627.

[6] Hartley G. H, Guillet J. E., Macromolecules 1 (1968) 165-170.

[7] Scott G., Atmospheric Oxidation and Antioxidants; Elsevier: New York 1965; Chapters 4 and 5, pp. 282-296.

[8] Al-Malaika S., Scott G., Degradation and Stabilisation of Polyolefins; Allen, N. S., Ed.; Applied Science Publishers: London, 1983; Chapters 6 and 7.

[9] Carlsson D. J., Garton A., Wiles D. M., DeVelopments in Polymer Stabilisation-1, Scott, G., Ed.; Applied Science Publishers: London 1979; pp. 220-221.

[10] Scott G., DeVelopments in Polymer Stabilisation-4, Scott, G., Ed.; Applied Science Publishers: London 1981; Chapter 1.

[11] Al-Malaika, S., Chakraborty K. B., Scott G., DeVelopments in Polymer Stabilisation-6, Scott, G., Ed.; Applied Science Publishers: London 1983; hapter 3.

[12] Grassie N, Scott G., Polymer Degradation and Stabilisation, Cambridge University Press: Cambridge 1985.

[13] Scott G.,. Atmospheric Oxidation and Antioxidants, 2nd ed.; Scott, G., Ed.; Elsevier: New York 1993; Vol. II, Chapters 3, 5, 8, and 9.

[14] Scott G., Antioxidants in chemistry, technology, medicine and nutrition, Albion Publishers: 1997; Chapters 1, 3, and 4.

[15] Gerald Scott, David M. Wiles, American Chemical Society 2(3), (2001) 615-622

[16] Scott G., Polymers and the EnVironment, Royal Society of Chemistry: Cambridge 1999; Chapter 2.

[17] Gilead D., Scott G., DeVelopments in Polymer Stabilisation-5, Scott, G., Ed.; Elsevier Applied Science Publishers: London 1982; Chapter 4.

[18] Scott G., Trends Polym. Sci. 5 (1997) 361-368.

[19] Gilead D., In Degradable Polymers: Principles and Applications, Scott, G., Gilead, D., Eds.; Chapman \& Hall: London 1995; Chapter 10.

[20] Fabbri A., Degradable Polymers: Principles and Applications, Scott, G., Gilead, D., Eds.; Chapman \& Hall: London 1995; Chapter 11.

[21] Manos G., Garforth A., Dwyer J., Ind. Eng. Chem. Res. 39 (2000) 1203. 
[22] Uemichi Y., Kashiwaya Y., Tsukidate M., Ayame A., Kanoh H., Bull. Chem. Soc. Japan 56 (1983) 2768.

[23] Audisio G., Bertini F., Beltrame P. L., Carniti P., Makromol. Chem.-Macromol. Symp. 57 (1992) 191.

[24] Ohkita H., Nishiyama R., Tochihara Y., Mizushima T., Kakuta N., Morioka Y., Ueno A., Namiki Y., Tanifuji S., Katoh H., Sunazyka H., Nakayama R., Kuroyanagi T., Ind. Eng. Chem. Res. 32 (1993) 3112.

[25] Ng S. H., Seoud H., Stanciulescu M., Sugimoto Y., Energy Fuels 9 (1995) 735.

[26] Shabtai J., Xiao X., Zmierczak W., Energy Fuels 11 (1997) 76.

[27] Arandes J. W.; Abajo I.; Lopez-Valerio D.; Fernandez I.; Azkoiti M. J.; Olazar M.; Bilbao J., Ind. Eng. Chem. Res. 36 (1997) 4523.

[28] Arguado J., Sotelo J. L., Serrano D. P., Calles J. A., Escola J. M., Energy Fuels 11 (1997) 1225.

[29] Sharratt P. N., Lin Y.-H., Garforth A. A., Dwyer J., Ind. Eng. Chem. Res. 36 (1997) 5118.

[30] Garforth A. A., Lin Y.-H., Sharratt P. N., Dwyer J., Appl. Catal. A 169 (1998) 331.

[31] George Manos, Arthur Garforth, John Dwyer, Ind. Eng. Chem. Res. 39 (2000) 1198-1202

[32] Plastics Recycling. In Uhlmann's Encyclopedia of Industrial Chemistry; VCH: Weinheim 1992; Vol. A21, pp. 64-68.

[33] Menges G., Fischer R., Lackner V., Int. Polym. Process. 7 (1992) 291-302.

[34] Pinto F., Franco C., Andre R., Tavares C., Dias M., Gulyurtlu I. Cabrita I., Fuel 82 (2003) 1967-1976.

[35] Gebauer M., Pfitzner R., Dommaschk V., Schneider J., Reuss J.; Wolf B., Meissner R., Wrackmeyer T., Patent DE 4329436, 1995.

[36] Tsuji T., Okajima S., Sasaki A., Masuda T., J. Chem. Eng. Japan 38 (2005) 859-864.

[37] Stefan Czernik, Richard J. French, Energy \& Fuels 20 (2006) 754-758. 